East African Medical Journal No. 8 August 2009

CHARACTERISTICS OF HIV-INFECTED CHILDREN SEEN IN WESTERN KENYA

W. M. Nyandiko, MBChB, MMed, Senior Lecturer, Department of Child Health and Paediatrics, Moi University School of Medicine, Eldoret, Kenya, USAID Academic Model Providing Access to Healthcare, Eldoret, Kenya, A. Mwangi, BSc, MSc, Biostatistician, USAID-Academic Model Providing Access to Healthcare, Eldoret, Kenya, Moi University, P.O. Box 4606, Eldoret, Kenya, S. O. Ayaya, MBChB, MMed, Associate Professor, E. C., Nabakwe, MBChB, MMed, Senior Lecturer, C. N. Tenge, MBChB, MMed, Lecturer, P. M. Gisore, MBChB, MMed, Lecturer, Department of Child Health and Paediatrics, Moi University School of Medicine, Eldoret, Kenya, USAID-Academic Model Providing Access to Healthcare, P.O. Box 4606, Eldoret, Kenya, E. Sang, BSc, Data Manager, USAID-Academic Model Providing Access to Healthcare, P.O. Box 4606, Eldoret, Kenya and R. C. Vreeman, MD, MS, Assistant Professor, Children's Health Services Research, Department of Paedriatrics, Indiana University School of Medicine Indianapolis, Indiana, USA

Request for reprints to: Dr. W. M. Nyandiko, Department of Child Health and Paediatrics, School of Medicine, Moi University, P.O. Box 4606, Eldoret, Kenya

\title{
CHARACTERISTICS OF HIV-INFECTED CHILDREN SEEN IN WESTERN KENYA
}

\author{
W. M. NYANDIKO, A. MWANGI, S. O. AYAYA, E. C. NABAKWE, C. N. TENGE, \\ P. M. GISORE and R. C. VREEMAN
}

\begin{abstract}
Objectives: To describe the characteristics and outcomes of children registered for care in a large HIV care programme in Western Kenya.

Design: A retrospective descriptive study.

Setting: USAID-AMPATH HIV clinics in health centres; district and sub-district hospitals; Moi Teaching and Referral Hospital in Western Kenya.

Subjects: HIV-infected children below age of 15 years seen in a network of 18 clinics in Western Kenya.

Interventions: Paediatric HIV diagnosis and care including treatment and prevention of opportunistic infections and provision of combination antiretroviral therapy (CART).

Main outcome measures: Diagnosis, clinical stage and immune status at enrollment and follow-up; hospitalisation and death. Descriptive statistical analyses and chi square tests were performed

Results: Four thousand and seventeen HIV-infected children seen between June 2002 and April 2008. Median age at enrollment was four years (0-14.2 years), 51\% girls, 25\% paternal orphans, $10 \%$ total orphans and $13 \%$ maternal orphans. At enrollment, $25 \%$ had weight-for-Age $Z$ scores $(W A Z) \geq-1$ and $21 \%$ had $W A Z$ scores $\leq 3$. Orphaned children had worse WAZ scores $(p=0.0001)$. Twenty five per cent of children were classified as WHO clinical stage 3 and 4, 56\% were WHO clinical stages 1 and 2 with $19 \%$ missing clinical staging at enrollment. Cough (25\%), gastroenteritis (21\%), fever (15\%), pneumonia (10\%) were the commonest presenting features. Twenty six per cent had been diagnosed with tuberculosis and only 25\% started on cotrimoxazole preventive therapy (CPT). Median CD4\% at enrollment was $16 \%(0-64 \%)$; latest recorded values were $22 \%(0-64)$. Sixty four per cent were on cART (cART + ), median age at start was 5.4 (014.4 years). The median initial CD4\% among cART+ was 13 (0-62) compared to 24 (0-64) for those not on ART (cART-). Median CD4\% for cART+ improved to $22 \%(0-59)$; whereas cART- was $23 \%(0-$ 64) at last appointment. During the period of follow-up, one fifth (19\%) of children on cART were lost to follow-up compared to slightly over one third $(37 \%)$ for those not on cART. Thirty four percent were hospitalised; $41 \%$ diagnosed with pneumonia. Six per cent of 4017 were confirmed dead.

Conclusions: HIV -infected children were enrolled in care early in childhood. Orphanhood was prevalent in these children as were gastroenteritis, fever, pneumonia and advanced immuno-suppression. Orphans were more likely to be severely malnourished. Only a quarter of children were put on cotrimoxazole preventive therapy. Children commenced on cART late but responded well to treatment. Loss to follow-up was less prevalent among those on cART.
\end{abstract}




\section{INTRODUCTION}

In the last two decades, the Acquired Immune DeficiencySyndrome(AIDS) epidemicthatiscaused by the Human Immunodeficiency Virus (HIV) has swept through sub-Saharan Africa with venom. According to the UNAIDS- www.unaids.org- there are 33.2 million people living with HIV, 22.5 million of them being in sub-Saharan Africa and 2.5 million being children under 15 years. In 2007 alone, there were 420,000 newly infected children worldwide with 330,000 children dying (1). In Kenya, over 1.3 million people are HIV infected, including over 150,000 children. The adult prevalence has apparently declined from a high of $15 \%$ in 1998 to a current low of $7.8 \%$ (2). Stabilised infection levels in this epidemic may be due to improved preventionbut also often result from rising death rates from AIDS which conceal a continuing high rate of new infections. There are over 1,100,000 orphans in Kenya due to parental HIV-infection and death. In 2005 alone, over 140,000 people died from HIV / AIDS in the country. HIV infection is one of the top five causes of mortality in Kenya and has greatly contributed to the under-five mortality raterising from 112 in 1998 to 120 per 1000 in 2004 (3).

HIV-related symptoms and signs are rarely present at birth, but develop over subsequent months or years (4). In about a quarter of infected children, HIV infection progresses rapidly to AIDS or death in the first year $(4,5)$. In the remainder, it progresses more slowly, with some HIV-infected children surviving child hood (6). The proportion of patients who have rapid progression of disease is higher in children than in adults. In the European collaborative study, mortality in HIV infected infants was $15 \%$, and mortality by the age of five years was $28 \%$, findings similar to those in the Italian study (6). According to the European experience, the annual rate of progression to AIDS decreases after the first year to approximately $6-8 \%$, as does the mortality rate $(5,6)$.

The morbidities of HIV-infected children may vary by geographical location. HIV -infected children in Africa frequently sufferfrom pneumonia, diarrhoea, bacteremia, failure to thrive and lymphadenopathy. In Cote d'Ivoire, among 338 consecutively admitted HIV-infected children, $26 \%$ had respiratory infections and $26 \%$ had malnutrition (7). In South Africa, among 48 children with vertically transmitted HIV infection followed for 26 months, $78 \%$ had diarrhoea, $76 \%$ pneumonia and $70 \%$ lymphadenopathy. Twenty five per cent died at a mean age of 10 months (but up to 48 months); the top four diagnoses at the time of death being dirrhoea, pneumonia, failure to thrive (FTT) and severe oral thrush (8). Mortality is also consistently higher among hospitalised children who are HIV-positive than those who are HIV-negative. The mortality rate among $354 \mathrm{HIV}$ positive children aged between six months and five years hospitalised in Zambia was 19\%, a rate significantly higher than the mortality rate of $9 \%$ among 912 hospitalised, HIV-negative children (9).

The effect of combination antiretroviral therapy (cART) on the disease progression and outcomes of HIV-infected children in Africa has not been wellstudied, in part because few children are on cART. At the end of 2006, the WHO estimated that 115,000 children had access to HIV treatment a coverage rate of about $15 \%$. In Kenya, only about 90,000 patients are on CART, out of an eligible population of over 750,000, with only 13,000 being children (10). Thus, data on African children's cART outcomes are just beginning to accumulate. In pooled data studying 392 children on CART in resource-limited settings including Africa, the median gain in $\mathrm{CD} 4 \%$ was 8.2 at 6 months ( $\mathrm{n}=48$, IQR: $4.8-12.6)$ and 10.2 at 12 months $(\mathrm{n}=12$, IQR: 6.6-16.3). The median Weight-for-Age Z score (WAZ) gain was +0.89 at 6 months and +0.92 at 12 months. Mortality over time was $7.8 \%$ (11). In a study in Western Kenya, children on cART had a good response irrespective of their orphan status, with the only remarkable finding being a lag in weight gain among the orphans on CART (12).

Moi University School of Medicine, in collaboration with Moi Teaching and Referral Hospital(MTRH), has recognised the pivotal role their institutions must play in Kenya's overall response to the threat presented by HIV-AIDS pandemic. These institutions, in partnership with Indiana University School of Medicine and USAID, established the USAID-Academic Model for the Prevention and Treatment of HIV-AIDS, now known as the USAIDAcademic Model Providing Access to Healthcare (USAID-AMPATH) (13). USAID-AMPATH embraces excellent patient care, education of patients, medical and nursing students, research and rural health centre participation. As Kenya implements anti-retroviral therapy in persons infected with the HIV virus, USAID-AMP A TH has provided care for over 89,000 patients, including over 16,000 children. The USAIDAMPATH cohort of patients provides an optimal setting in which to describe HIV-related outcomes for Paedriatic patients in East Africa.

We need to examine the demographic and clinical characteristics, morbidities and outcomes for HIV-infected children in East Africa. Such studies will inform strategic protocols and improve the efficacy of HIV care and management. Describing HIV-related characteristics and outcomes for our cohort of HIV -infected children will not only benefit the thousands of children followed by the USAID-AMPATH programme, but will also provide important information for Kenya, the East African region, and beyond.

Themain objectiveof thestudy was to describe the demographic and clinical characteristics; diagnoses; 
treatment and follow-up outcomes among a cohort of HIV-infected children in care at a large HIV care programme in Western Kenya.

\section{MATERIALS AND METHODS}

Study design: This was a retrospective descriptive study. We used prospectively collected de-identified data from the computerised medical records of HIV -infected paedriatic patients treated in the USAIDAMPATH clinical care system in Western Kenya. The study was approved by the Institutional Research and Ethics Committee of the Moi University School of Medicine and Moi Teaching and Referral Hospital (Eldoret, Kenya) and the Institutional Review Board of the Indiana University School of Medicine (Indianapolis, IN).

Study site: USAID-AMPATH operates an urban referral clinic at the Moi Teaching and Referral Hospital (MTRH) in Eldoret, Kenya, a national tertiary referral hospital serving a catchments area of approximately 13 million people. Comprehensive HIV care services for children are also provided at 17 outlying outpatient clinics (Mosoriot, Turbo, Burnt Forest, Amukura, Naitiri, Chulaimbo, Webuye, Teso, Kitale, Kapenguria, Mt. Elgon, Hen, Kabarnet, Busia, Port Victoria, Uasin-Gishu and Khuyangu), located within district hospitals and health centres throughout Western Kenya. HIV care is provided by paeditricians, medical officers and clinical officers trained and mentored within AMPATH.

Study population: Eligible patients included any child seen in any of 18 USAID-AMPATH clinics between June 2002 and April 2008 who was HIV-infected and less than 15 years of age.

Clinical procedures: Throughout the period of the study, clinicians followed detailed, locally developed protocols consistent with World Health Organisation guidelines. HIV infection was documented by DNAPCR(Amplicor, Roche, Basel, Switzerland) forchildren $<18$ months and by two parallel HIV rapid ELISA tests using Determine and Unigold kits for children $>18$ months. cART was initiated for any children $<6$ years of age with a CD4 cell percentage of $<15 \%$, for any child $>6$ years of age with a CD 4 count $<200$ cells $/ \mathrm{mm}^{3}$, and for all children with WHO clinical stages 3 or 4 or CDC stage $C$ disease. The standard initial cART regimens used were zidovudine/lamivudine/nevirapine for those weighing $<10 \mathrm{~kg}$ or stavudinel/amivudine/ nevirapine for those weighing $>10 \mathrm{~kg}$. All patients were started on cotrimoxazole preventive therapy if below the age of six years. Those above six years of age with a CD4 \% of less than $25 \%$ and those with WHO stages 3 or 4 were also put on cotrimoxazole. Children started on cART were seen two weeks after initiation of therapy, and then every month thereafter. During these visits, patients undergo clinical assessments, including assessment of family and social characteristics, nutritional status, the degree of immuno-suppression, current or chronic infections, dietary intake, drug/treatment history and adherence history. Laboratory investigations included routine assessment of CD4\% every six months and investigations to evaluate haemoglobin level, liver, kidney functions every three months. Tests to evaluate for toxicities were performed as necessary. Loss to follow-up was defined as missing clinic appointment for more than six months for those not on CART and more than three months for those on CART. An orphan was defined as a child who had lost either both or one of the parents. Nutritional status was defined using the following categories: a weight-for-age $Z$ score (WAZ) of $\geq 1$ indicated normal nutritional status, a WAZ score of -1 to -2 indicated mild malnutrition, a WAZ score of -2 to -3 indicated moderate malnutrition, and a WAZ score of $\leq 3$ indicated severe malnutrition.

Data collection and management: Clinicians completed standard initial and return encounter forms at all USAID-AMPATH clinic visits for the children ( $h t t p: / /$ amrs.iu-Kenya.org/download/forms). Theinitialencounter form included standard demographic; birth and prevention of mother to child transmission prophylaxis history; dietary intake; social; physical; examination; laboratory data and medications provided including antiretroviral drugs and opportunistic infection prophylaxis. At each subsequent clinical encounter, follow-up data were collected on inter-current symptoms, medication adherence, new diagnoses, laboratory data, and modifications in medication regimens. Dedicated data entry clerks entered this information into the ambulatory USAID-AMPATH Medical Record System with data entry validated by random review of $10 \%$ of the data entered. The medical records system was MS Access-based (Microsoft Corporation, Redmond,WA) until2006, and currently uses MySQL based database.

Statistical methods: Descriptive statistics such as mean, standard deviation, median and range were used for the continuous variables while frequency listings were used for categorical variables. The chi-square test was used to assess any association between a categorical variable and each of the independent variables, where the cell count were below 10 the Fishers' exact test was used in 2 by 2 tables. Plots were used to explore the trends of the CD4\% of children over time, and this was done both overall and by orphan status, since orphan status was hypothesized to have an effect on the trend. All statistical analyses were performed using SAS Institute version 9.1. A p-value of less than 0.05 was considered significant in all analyses. 


\section{RESULTS}

Data were available for 4017 HIV-infected children who were seen in the USAID-AMPATH outpatient HIV clinics from June 2002 to April 2008. The majority of patients were enrolled at the outpatient HIV clinic at the urban outpatient clinic of Moi Teaching and Referral Hospital (33\%) with the remaining patients enrolled at district hospitals and rural health centres (Table 1). The median age at enrollment was 4.5 years (range: 0 years-14.2 years), with the median duration of follow-up being 15 months (range: 0.00 74 months). At enrollment, $10 \%$ of the children were less than five months old, almost $50 \%$ were between five months and five years old, and $40 \%$ were older than five years. About half of the children were girls $(51 \%)$. A total of $59 \%$ were orphaned at enrollment: $25 \%$ being paternal orphans, $13 \%$ maternal and $11 \%$ total orphans. The predominant ethnic group of the children was Luhya (32\%) with the rest being Kalenjin $(27 \%)$, Luo (20\%), Kikuyu (10\%) and others. At the time of cART initiation, more than half of the children on cART were brought to the clinic by their mothers $(52 \%), 14 \%$ by their grandparents, $11.5 \%$ by an auntie or uncle and only $8 \%$ by their fathers. The majority of children had less than two siblings (53\%), with only $14 \%$ having five or more siblings.

Table 1

Demographic characteristics of children

\begin{tabular}{lll}
\hline & No. & $(\%)$ \\
\hline Gender & & \\
Male & 1959 & 48.78 \\
Female & 2057 & 51.22 \\
Clinic & & \\
MTRH & 1307 & 32.54 \\
MRHC & 343 & 8.54 \\
Turbo & 236 & 5.88 \\
Burnt Forest & 219 & 5.45 \\
Chulaimbo & 353 & 8.79 \\
Webuye & 223 & 5.55 \\
Kitale & 345 & 8.59 \\
Busia & 158 & 3.93 \\
Distant clinics & 833 & 20.74 \\
Orphaned status & & \\
Paternal & 995 & 24.77 \\
Maternal & 536 & 13.34 \\
Total & 428 & 10.65 \\
Non orphaned & 1145 & 22.73 \\
Unknown & 913 & 28.50 \\
Confirmed dead? & & \\
Yes & 224 & 5.58 \\
No & 3793 & 94.42 \\
& &
\end{tabular}

Ever On ARVs?

$\begin{array}{lll}\text { Yes } & 2576 & 64.13 \\ \text { No } & 1441 & 35.87 \\ \text { Child attended school } & & \\ \text { Yes } & 882 & 41.25 \\ \text { No } & 247 & 11.55 \\ \text { Non-applicable } & 1009 & 47.19 \\ \text { Missing } & 1879 & \end{array}$

Clinical status at presentation: The majority $(78 \%)$ of the children over nine months of age had completed their immunisations as defined by the Kenya Expanded Programme of Immunization (KEPI) schedule. More than a quarter $(25 \%)$ of the patients had normal WAZ scores at enrollment, another one quarter (26\%) had mild malnutrition, $26 \%$ had moderate malnutrition, and $22 \%$ were severely malnourished (Table 2).

Table 2

Anthropometric measures $(n=4017)$

\begin{tabular}{lcl}
\hline & No. & $(\%)$ \\
\hline Age at enrolment in & categories \\
$<5$ months & 410 & 10.60 \\
5 months- 1 year & 328 & 8.48 \\
1 to 5 years & 1549 & 40.04 \\
$5-12$ years & 1462 & 37.79 \\
$12-15$ years & 120 & 3.10 \\
Age at start of ART in categories & \\
$<1$ year & 146 & 5.68 \\
1 to 5 years & 1050 & 40.87 \\
$5-12$ years & 1248 & 48.58 \\
$12-15$ years & 125 & 4.87 \\
WAZ score closest to enrolment $(\mathrm{n}=3724)$ \\
$\leq 3$ & 816 & 21.91 \\
-3 to -2 & 982 & 26.37 \\
2 to -1 & 990 & 26.58 \\
$\geq 1$ & 936 & 25.13 \\
Missing & 170 & \\
WAZ score at last & & \\
appointment (n=3765) & & \\
$\leq 3$ & 535 & 14.21 \\
-3 to -2 & 843 & 22.39 \\
-2 to -1 & 1220 & 32.40 \\
$\geq 1$ & 1167 & 31.00 \\
Missing & 129 & \\
\hline
\end{tabular}

There was a statistically significant difference between the nutritional status of non-orphaned children compared to the orphaned children at enrollment $(p=0.0001)$, with orphaned children having more malnutrition (Table 3). 
Table 3

Comparing nutritional status by orphan status

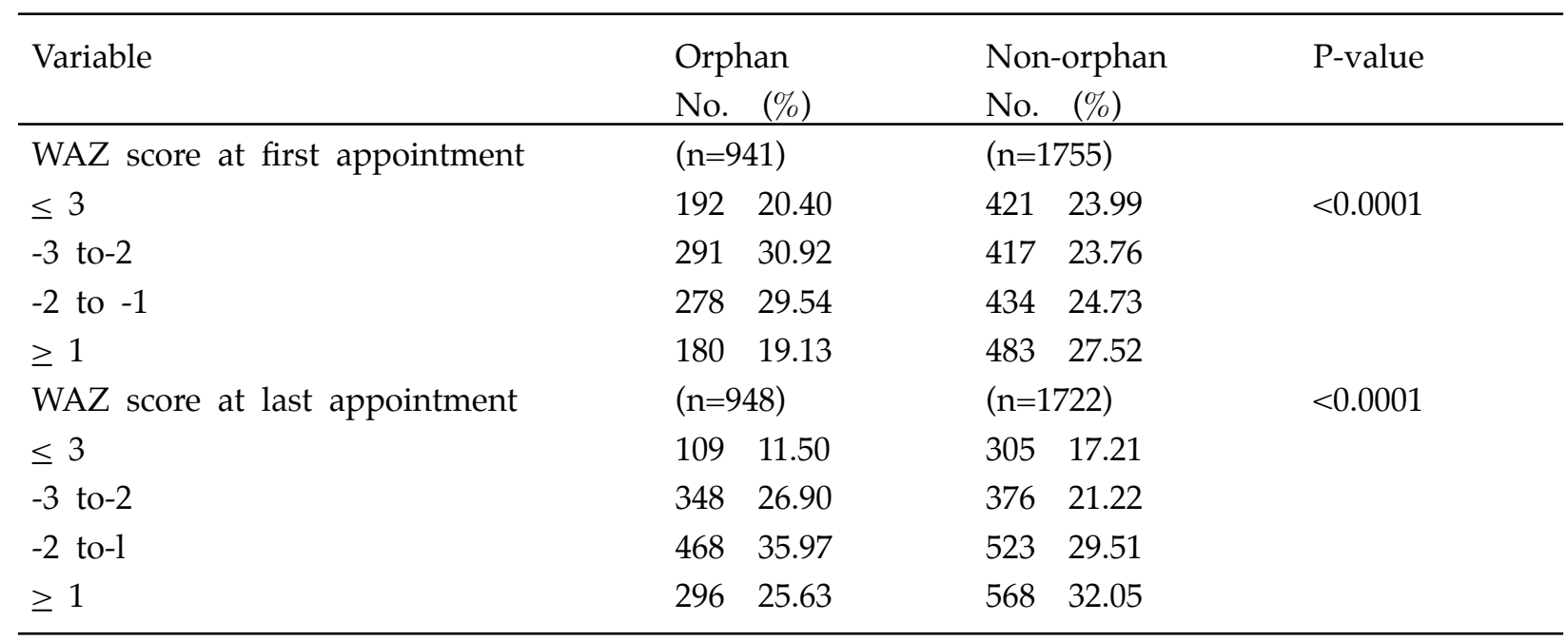

More than two thirds of the children were either asymptomatic or mildly symptomatic at enrollment $(56 \%)$, with only $26 \%$ being in WHO stages 3 and $18 \%$ inWHOstage4(Table4). Themostcommon presenting symptoms and diagnoses at enrollment were rash/ dermatitis (32\%), cough (25\%), gastroenteritis (21\%), fever $(15 \%)$, pneumonia $(10 \%)$, otitis media $(8 \%)$, oral thrush $(8 \%)$, failure to thrive $(6 \%)$, and delayed milestones $(2.5 \%)$. Slightly more than one quarter had been diagnosed with tuberculosis (TB) at some time point either before or after enrollment. Only one quarter $(24 \%)$ had ever been on cotrimoxazole for pneumocystis carinii prophylaxis (Table 4).

Table 4

Clinical diagnoses and signs and symptoms $(n=4017)$

\begin{tabular}{lll}
\hline & No. & $(\%)$ \\
\hline WHO staging at & & \\
enrolment $(\mathrm{n}=3844)$ & & \\
Stage 1 & 957 & 24.90 \\
Stage 2 & 1193 & 31.04 \\
Stage 3 & 998 & 25.96 \\
Stage 4 & 696 & 18.11 \\
Missing & 173 & \\
WHO staging at last & & \\
appointment $(\mathrm{n}=3844)$ & & \\
Stage 1 & 695 & 18.08 \\
Stage 2 & 895 & 23.28 \\
Stage 3 & 964 & 25.08 \\
Stage 4 & 1290 & 33.56 \\
Missing & 173 & \\
Develop TB? (n=4017) & & \\
Yes & 1083 & 26.96 \\
No & 2934 & 73.04
\end{tabular}

Develop TB? $(\mathrm{n}=1083)$

At enrolment 215

19.85

After enrolment $\quad 868 \quad 80.15$

Develop TB? $(\mathrm{n}=1083)$

$\begin{array}{lll}\text { After ARV } & 174 & 16.07 \\ \text { Before ARV } & 559 & 51.62 \\ \text { Never on ARV } & 350 & 32.32 \\ \text { Hospitalisation } & & \\ \text { Yes } & 778 & 19.38 \\ \text { On septrin } & 978 & 24\end{array}$

Over 9 months $(\mathrm{n}=3702)$

Completed immunisation

2898

Table 5

Laboratory tests

CD4\% closest to enrolment $(n=2975)$

$$
\begin{array}{ll}
\text { Mean (sd) } & 17.49(10.96) \\
\text { Median (range) } & 16.00(0,64)
\end{array}
$$

$\mathrm{CD} 4 \%$ at last appointment $(\mathrm{n}=3273)$

$$
\begin{array}{ll}
\text { Mean (sd) } & 22.08(10.41) \\
\text { Median (range) } & 22.00(0.64)
\end{array}
$$

CD4 count closest to enrollment $(n=3275)$

$$
\begin{array}{ll}
\text { Mean (sd) } & 639.56(587.58) \\
\text { Median (range) } & 484(0.4337)
\end{array}
$$

CD4 count at last appointment $(n=3317)$

$$
\begin{array}{ll}
\text { Mean (sd) } & 815(588) \\
\text { Median (range) } & 703(0.4262)
\end{array}
$$

WBC at closest to enrolment $(n=984)$

Mean (sd)

7619 (4247)

Median (range) 
$\mathrm{Hgb}$ at closest to enrolment $(\mathrm{n}=2851)$

$\begin{array}{cl}\text { Mean (sd) } & 14.55(226) \\ \text { Median (range) } & 10.40 \\ & (0.10 .70,12100) \\ \text { ALC at closest to enrolment } & (\mathrm{n}=578) \\ \text { Mean (sd) } & 3829.58 \\ & (2898.35) \\ \text { Median (range) } & 3310(1.58,4600)\end{array}$

Clinical and immunologic course: By the time of the last appointment in the database, there was an improvement in nutritional status for most children (Table 3). Orphaned children continued to be more likely to be malnourished (were time, with significantly lower WAZ scores at the most recent assessment of nutritional status $(p=0.0001)$.

Of the 1043 diagnosed with TB, the majority $(80 \%)$ were diagnosed after enrollment. The majority of the children who developed TB were diagnosed with TB prior to initiation of cART (52\%) compared to $16 \%$ who developed TB while on cART.

The median CD4 count and percentages closest to enrollment were 484 (range: 1-4337) and 16\% (range: 0-64\%) respectively (Table 6).

The median CD4 count and percentage increased slightly when examining the most recent clinical measurement, 703 (range 1-4262) and 22\% (range $0-64$ ) respectively (Figures 1 and 2). Orphan status did not affect these trends. (Figures 3 and 4 ).

\section{Figure 1}

CD4 percent profile for children on ART

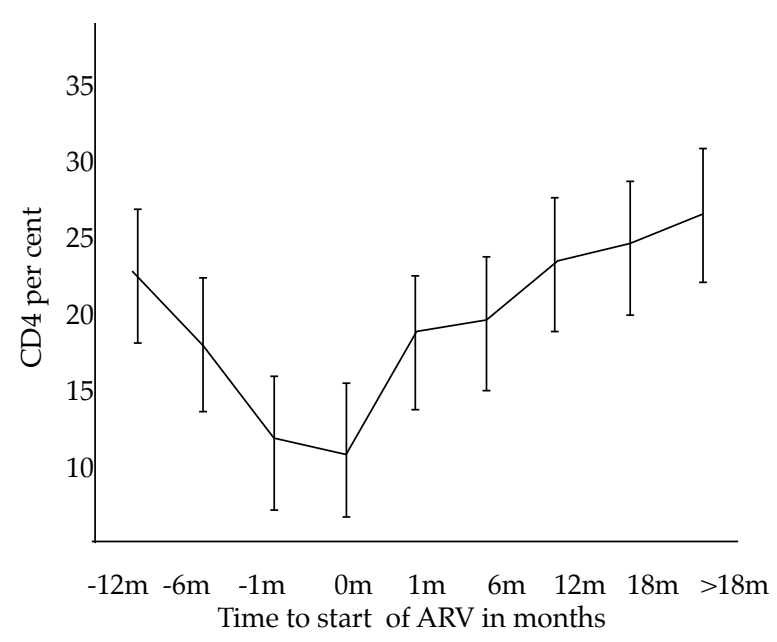

Figure 2

CD4 percent profile for children not on ART

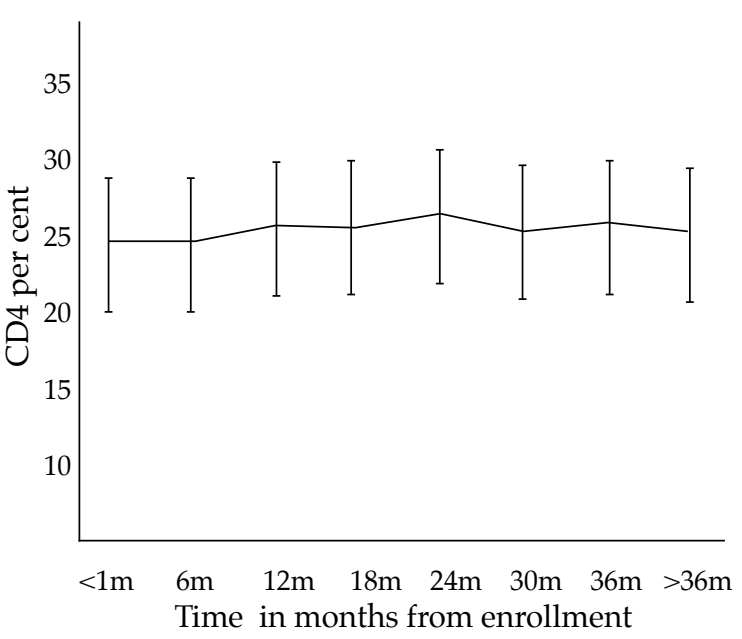

Anti-retroviral therapy and opportunistic infections prophylaxis status: About two thirds (64\%) of all HIV -infected children were on CART during the period of the study. The median age at start of the cART was 5.3 years (range: $0-14.4$ years). Only $6 \%$ of the children on ART were below one year of age, $41 \%$ between one and five years, and more than half (52\%) above five years.

The children started on cART had a median CD4 count and percent of 375 (range 1-4202) and $13 \%$ (range 0-62\%) respectively closest to enrollment. Those not on ART had higher counts of 814 (range $0-4331$ ) and $24 \%$ (range $0-64 \%$ ) respectively closest to enrollment (Table 6). The median CD4 counts increased at the last appointment for those on cART to 671 (range 0-4262) and 22\% (range 059). However, these values declined slightly in those not on cART to 787 (range 0-3940) and 23 (range 0-64) respectively. (Figures 1 and 2) Among those on cART, orphaned children tended to have a lower latest recorded CD $4 \%$ compared to the non-orphaned (Figure 3 ). The trends of CD4 percent did not vary much between the different age categories (Figure 5). 
Figure 3

Median CD4 percent for those on ART by orphan status

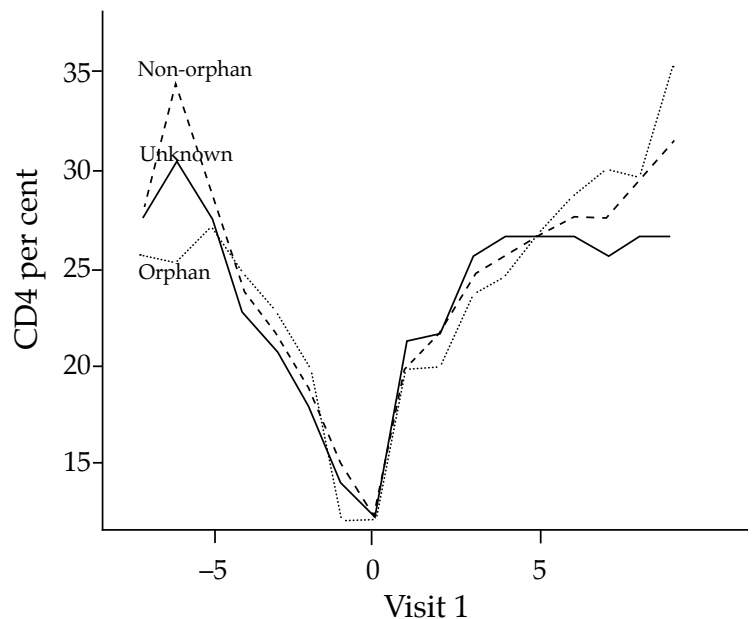

Figure 4

Median CD4 percent for those not on ART by orphan status

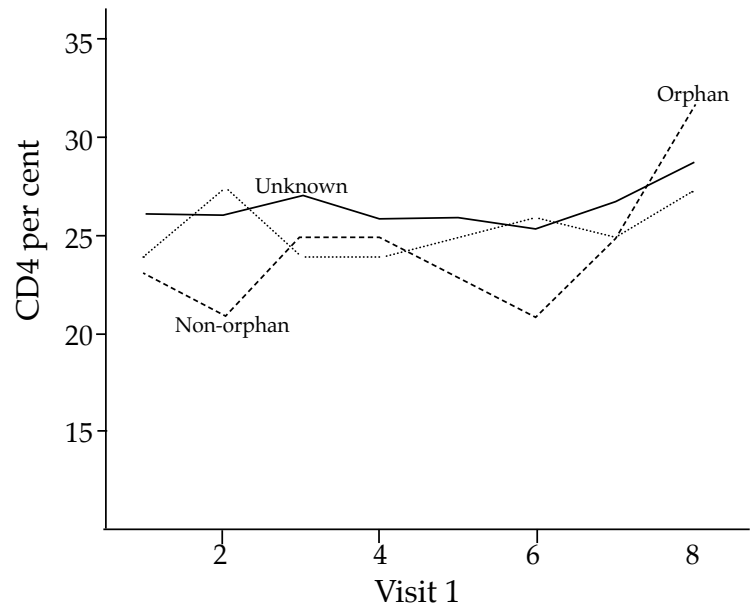

Figure 5

Median CD4 percent for children on $C A R T$ with age

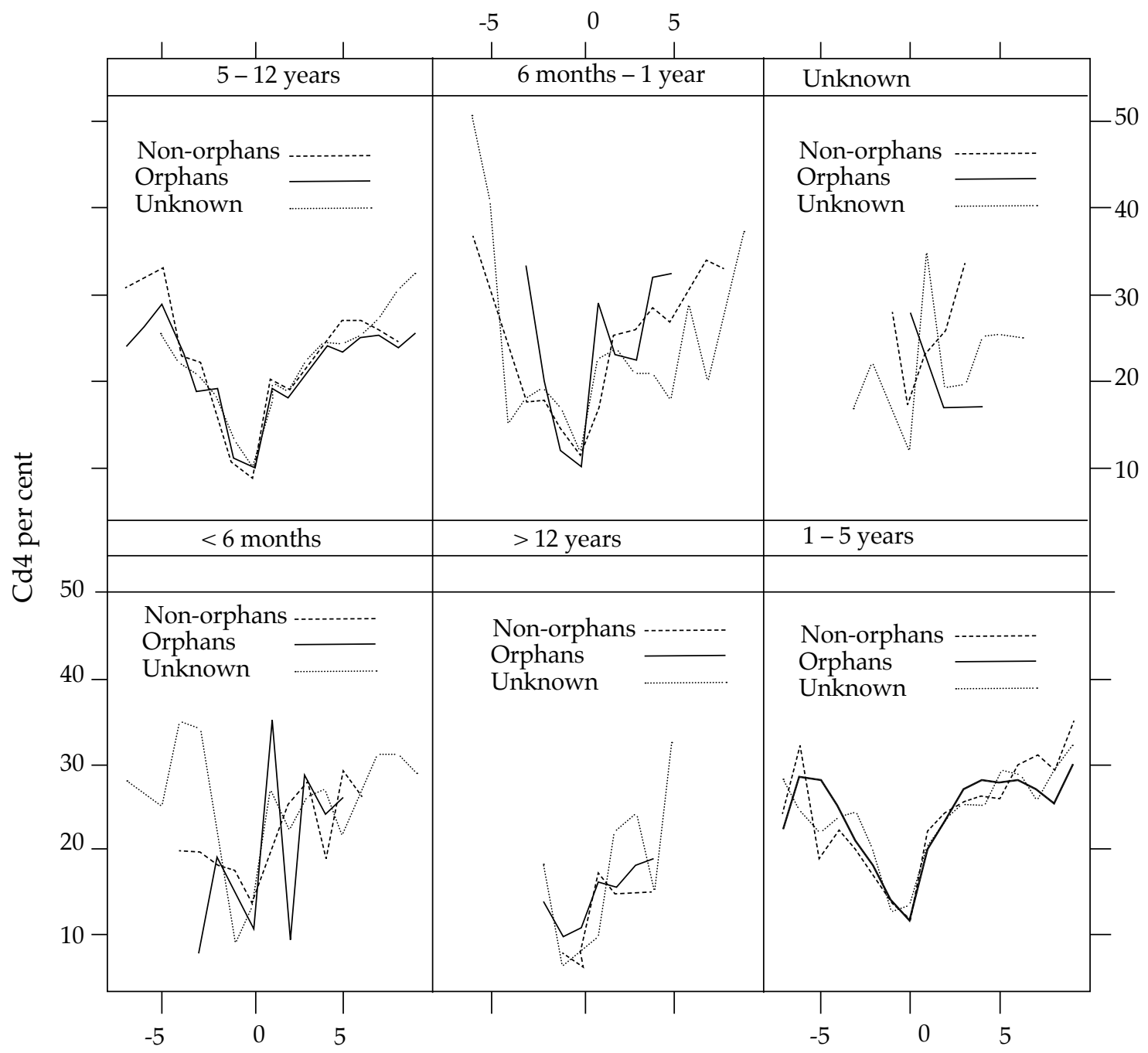

Visits 
Table 6

CD4 by ARV status

\begin{tabular}{lll}
\hline & Ever on ARV & Not on ARV \\
\hline CD4\% closest to enrolment & $(\mathrm{n}=2107)$ & $(\mathrm{n}=868)$ \\
Mean (sd) & $14.66(9.84)$ & $24.38(10.48)$ \\
Median( range) & $13.00(0.62)$ & $24(0.64)$ \\
CD4\% at last appointment & $(\mathrm{n}=2380)$ & $(\mathrm{n}=893)$ \\
Mean (sd) & $21.66(10.64)$ & $23.20(9.70)$ \\
Median (range) & $22(0,59.00)$ & $23.0(0.64)$ \\
CD4 count closest to enrolment & $(\mathrm{n}=2380)$ & $(\mathrm{n}=900)$ \\
Mean (sd) & $505.54(475.44)$ & $992.93(698.12)$ \\
Median( range) & $375(1.4202)$ & $814(0.4331)$ \\
CD4 count at last appointment & $(\mathrm{n}=2406)$ & $(\mathrm{n}=911)$ \\
Mean (sd) & $768.77(558.79)$ & $935.01(646.57)$ \\
Median(range) & $671.5(1.4262)$ & $787(0.3940)$ \\
\hline
\end{tabular}

Follow-upand admission characteristics: Aquarter(26\%) of the children were lost to follow-up during the duration of follow-up, with $19 \%$ of those on cART being lost to follow-up compared to slightly over one third (37\%) for those not on cART. Only $6 \%$ were confirmed dead after enrollment.

About one fifth of the children (19\%) were ever admitted after enrollment to various hospitals in the vicinity of AMPATH clinics during the study period. Ninety one patients were admitted to the biggest and referral facility in the programme, MTRH. Documentation for admission to other peripheral health facilities was incomplete and was not included. The most common reasons for admission at MTRH were pneumonia $(n=60)$, malaria $(n=22)$, pulmonary tuberculosis $(n=14)$, meningitis $(n=9)$, dehydration $(n=9)$ and malnutrition $(\mathrm{n}=5)$. Of those admitted in MTRH, 24 died and the rest were discharged. One third (32\%) of all those admitted had ever been on cART.

\section{DISCUSSION}

Inthis retrospectivestudy, wedescribethecharacteristics of alarge cohort of HIV infected childrenin sub-Saharan Africa. The most common presenting symptoms and diagnoses in this cohort compares well with other African cohorts $(8,9)$. Skin rash; chronic or recurrent cough probably associated with tuberculosis, lymphoid interstitial pneumonitis and upper respiratory tract infections; gastroenteritis and oral thrash formed some of the common presenting features. Interestingly, only $26 \%$ of the children seen in this cohort were diagnosed with tuberculosis before and after enrollment. This is low compared to estimated rates of TB in other children populations in Africa (9). However, this may be an indication that we are under-diagnosing tuberculosis in children, a well recognised challenge in resource limited settings where diagnostic capabilities are still limited and criteria for diagnosing TB still not well validated in the background of HIV infection (14).

Amajority of the children presented at a relatively good WHO clinical stage, emphasizing the fact that our local population may be heeding the call by health personnel, community mobilisers and media for the affected families to present early for testing and care. In addition, this means that the children in our cohort are identified early for care. In contrast to early literature describing late presentation of African children (7-9), we are only seeing about $40 \%$ in WHO stages 3 and 4 at presentation. The rapid progression of HIV in children may be explained by the level of maturity of the immune system at the time of HIV acquisition. Infants with HIV are also exposed to primary infections and opportunistic organisms which is different from the reactivation of such infections that occurs in adults.

Early enrollment to paedriatic HIV care will translate to early treatment of opportunistic infections and early initiation on ART and therefore better survival $(15,16)$. Wehowevernoted alow uptakeof cotrimoxazole preventive therapy. This may be explained by the fact that almost half of the children have a good clinical and immunological status and therefore did not qualify for CPT.Wehavehowever alsonoted alack of compliance to the protocols by some clinicians. Thisis being addressed by retraining and mentoring of theclinicians. Wearealso in the process of introducing electronically generated reminders to clinicians to initiate all eligible children on CPT. This will definitely help reduce the morbidity and mortality from common conditions prevalent in this population of children as has been described in Zambian children (17).

In this cohort, the majority of children receiving care in the comprehensive clinics in Western Kenya are the under-fives, indicating the increased ability of prevention of mother to child service and other entry 
points in identifying the infants early and starting them on co-trimoxazole and follow-up. This might explain the relatively low crude mortality in this cohort. Early infant diagnosis is known to prevent morbidity and mortality as has been described in studies in South Africa and Uganda $(15,19)$. In the AMPATH programme, the pMTCT programme was ramped up in 2002-2004 leading to early clinic attendance in index children and their siblings (13). We therefore demonstrate that it is possible to diagnose these childrenearly despite theoften reported delays in early diagnosis in resource limited settings. The use of all points of entry including ante natal clinic, outpatient clinics, TB clinics and wards for paedriatic diagnosis is not reported in many care settings. In Uganda and South Africa $(18,19)$ efforts in ANC and outpatient clinics reported improvements in the rate of early diagnosis comparable to what we find in this study. More resources and targets need and can be set aside to achieve this lifesaving objective in programmes in low income countries.

The immunologic status of the children was also relatively good unlike the findings of the adult population in the same care and in other studies in Africa (20). Immunologic status was lower at enrollment for those started on ART. The median CD4 count and percentage was lower when we looked at the most recent counts for those not on ART and, as expected, was higher in those on ART in recent counts. This suggests the success of ART in this cohort, and matches the previous analyses of an earlier cohort (12) and other African cohorts (20). The trends of CD4 percent in different age categories did not vary much (Figure 5). One would have expected a more rapid decline for thosebelow one year of age. This is perhaps explained by the consistent follow-up, prevention and treatment of opportunistic diseases in these children thus maintaining a relatively good CD4 percent.

The nutritional status of the children was relatively good at enrollment. It was significantly lower at enrollment for those who subsequently began ART. The status was also significantly lower among orphaned children compared with the non-orphaned, a feature that has been noted in an earlier cohort in the same programme on ART (12). In that study, the orphaned children seemed to lag in improvement of their nutritional status compared to the non-orphaned after 100 weeks of follow-up. In the current analysis, nutritional status improved in both groups albeit a small lag of the latest values for the orphaned on CART, which may reflect the impact of nutritional support for families that is now provided in this programme (13). In this nutritional programme, children with malnutrition or food insecurity as assessed by the nurses or nutritional assistants ateach visit are initiated on food rations that include pulses and oil (provided by World Food Program) and vegetables (provided from USAID-AMPATH demonstration farms) until there is improvement in the nutritional status and food security situation. This is an important finding that requires more investigation on the effect of good nutrition in delaying initiation of ART in children.

The median haemoglobin level at enrollment in this cohort was relatively high at $10 \mathrm{gm} / \mathrm{dl}$. This is a reassuring finding in such a young population, particularly since a significant number of the children are using zidovidune, a potential bone marrow suppressant. In addition, the median white cell count and absolute lymphocyte counts were relatively high, though lower in the group that was started on ART. There were no significant differences in the absolute lymphocyte counts from different geographical and therefore ethnic sites, as contrast to findings in the adult population in the same programme (unpublished data, under review at EAMJ).

Less than one quarter of the patients were ever admitted to hospital. The most common reasons for admission of the cohortseen atMTRHwere pneumonia, gastroenteritis, malaria, pulmonary tuberculosis and meningitis. These findings are similar to those in other African countries $(8,9,20,21)$. Mortality was low in this cohort, reflecting relatively good care in a resourceconstrained setting. However, there is a high loss to follow-up, and it is difficult to say whether some of these might be unreported deaths. The children on cART are less likely to be lost to follow-up and this is probably evidence of sustained education, support and follow-up that is accorded to children on cART by the caregivers and care providers. The difficulty associated with inability to trace our patients is a common feature in Africa where homes do not have well defined location. This is however being addressed in an outreach programme within USAID-AMPATH, and we hope to have less loss to follow-up and better data on mortality form this cohort in the future.

The majority are brought to the clinic by their mothers as is cultural in African countries. However, the grandparents, aunts and uncles also playa key role in bringing HIV infected children for care in those that areorphaned. This has been described in other African cohorts where the extended families have been able to lessen the burden that the orphans would otherwise face (22). More than two thirds of the children seen in this cohort are orphaned, with more than $10 \%$ having both parents' dead. This is consistent with the high prevalence of orphan-hood within Kenya. Other studies in African countries report higher rates of orphan-hood (22). Within this population, more children have lost their fathers than their mothers. This may reflect that women seek healthcare earlier than men as reported in WHObulletins. In addition, the higher prevalence of paternal orphans may spring from earlier HIV infection, progression, and death among men compared to women (22).

This cohort describes findings in children that have access to a comprehensive package including nutritional support, micro-enterprise programmes to families to facilitate income generation, and free drugs, including ART and drugs for opportunistic infections. It may therefore not be representative, 
at least in terms of disease progression in other resource-limited settings, including those run by the Kenyan Ministry of Health. In addition, the high rate of LTFU may affect the findings of this study.

In conclusion, we note that HIV -infected children were enrolled in care early in childhood. Orphanhood was prevalent in these children as were gastroenteritis, fever, pneumonia and advanced immuno-suppression. Orphans were more likely to be severely malnourished. Only a quarter of children were put on contimoxazole preventive therapy. Children commenced on cART late but responded to treatment well. Loss to follow-up was less prevalent among those on cART.

We recommend long term follow-up of children in this and other similar settings to determine the natural progression for those on ART and those not on ART. The nutritional status of orphaned children needs to be assessed more aggressively and managed early. Efforts to increase uptake of cotrimoxazole preventive therapy and decrease the loss to followup need to be intensified.

\section{ACKNOWLEDGEMENTS}

This research was supported in part by a grant to the USAID-AMPATH Partnership from the United States Agency for International Development as part of the President's Emergency Plan for AIDS Relief (PEPFAR). The authors give special thanks to the families and to the health care providers of AMPATH, including the nurses, clinicians, and pharmacy staff, all of who worked tirelessly to ensure that the children of Western Kenya receive the medications they need. In particular, we would like to thank Dr. L. Aluoch (Mrs), Dr. I. Marete and the other members of the AMPATH Paediatric Research Working Group. We also wish to thank IREC, the Director of MTRH and the Dean Moi University School of Medicine for allowing us to collect data and conduct research on the patients seen.

\section{REFERENCES}

1. UNAIDS. Report on the globalHIV / AIDS epidemic Global report. Geneva: World Health Organization, 2007.

2. Kenya AIDS indicator survey 2007. Preliminary report. Nairobi, Kenya.

3. World Health Organization. Mortality country fact sheet 2006. http://www . who.int/whosis/mort/profiles/ mort-afro-ken - kenya.pdf.

4. European Collaborative Study. Children born to women with HIV -1 infection: natural history and risk of transmission. Lancet. 1991; 337: 253-260.

5. The European Collaborative Study. Natural history of vertically acquired HIV-1 infection. Paediatrics. 1994; 94: 815-819.

6. Italian register for HIV infection in children. Features of children perinatally infected with HIV -1 surviving longer than 5 years. Lancet. 1994; 343: 191-195.
7. Vetter, K.M., Djomand, G., Zadi, F., et al. Clinical spectrum HIV disease in a west African city. Project RETRO-CI. Pediatr. Infect. Dis. J. 1996;15: 438-442.

8. Bobat, R., Moddley, D., Coutsoudis, A., et al. The early natural history of vertically transmitted HIV -1 infection in African children from Durban, South Africa. Ann. Trop. Paediatr. 1998; 18: 187-196.

9. Chintu, C., Luo, C., Bhat, G., et al. Impact of the HIV-1 on common paediatric illnesses in Zambia. J. Trop. Pediatr. 2000;137: 78-84.

10. Kimani, F., Director of Medical Services, MOHKenya. Health: Lack of ARV's a risk to 10,000 Children. Daily Nation Newspaper Friday 19th 2008, pg 6. www.nation.co.ke

11. Humblet, P., Calmy, A., Pinoges, L., et al. Offering HAART to children in resource poor settings: The experience of Medicins Sans frontiers. Abstract in XV International AIDS conf. Vol 1, pg 337.

12. Nyandiko, W.M., Ayaya, S.O., Nabakwe, E., et al. Outcomes of HIV-infected children on anti-retroviral therapy at AM PATH HIV clinics in Western Kenya. J. AIDS. 2006; 43: 418-425.

13. Einterz, R.M., Kimaiyo, S., Mengech, H.N.K. et al. Responding to the HIV pandemic: the power of an academic medical partnership. Academic Med. 2007; 82: 812-818.

14. Hesseling, A.C., Schaaf,H.S., Gie, R.P.,Starke, J.R. and Beyers, N. A critical review of diagnostic approaches used in diagnosis of childhood tuberculosis. Inter. J. Tuberc. Lung. Dis. 2002; 21: 1038-1045.

15. Mukadi, Y.D., Wiktor, S.Z., Coulibaly, I.M., et al. Impact of HIV infection on the development, clinical presentation, and outcome of tuberculosis among children in Abidjan, Cote d'Ivoire. AIDS. 1997; 11:1151-1158.

16. Eley, B., Nutall, J., Davis, M. and Smith, L. Initial experience of a public sector antiretrovira1 treatment program for HIV infected children and their infected parents. S. Afr. Med. J. 2004; 94: 643-646.

17. Chintu, C., Bhat, G. J., Walker, A.S. et al. Cotrimoxazole as prophylaxis against opportunistic infections in HIV-infected Zambian children (CHAP): a double-blind randomised placebo-controlled trial. Lancet. 2004; 364: 1865-1871.

18. Thior, I., Lockman, S., Smeaton, L. M. and Shapiro, L. R. Breastfeeding plus infant zidovudine prophylaxis for 6 months vs formula feeding plus infant zidovudine for 1 month to reduce mother-tochild HIV Transmission in Botswana :A randomized trial: The Mashi Study. JAMA. 2006; 296: 794.

19. Palombia, L., Marazzib, M. C. and Voetbergc, A. Treatment acceleration program and the experience of the DREAM program in prevention of mother- tochild transmission of HIV. AIDS. 2007; 21 (suppI 4): 65-71.

20. Nabukeera-Barungi, N.,Kalyesubula, I., Kekitiinwa,A., et al. Adherence to antiretroviral therapy in children attending Mulago Hospital, Kampala. Ann. Trop. Paediatr. 2007; 27: 123- 131.

21. Bolton-Moore, C., Mwangelwa, M., Cantrell, R., et al. Clinical outcomes and CD4 response in children receiving ART at primary Health care facilities in Zambia. JAMA. 2007;298/16: 1888-1899.

22. Kamali, A., Seeley, J.A., Nunn, A.J., et al. The orphan problem: experience of a sub-Saharan African rural population in the AIDS epidemic. AIDS care. 1996; 8:509-515. 